\title{
CATEGORIES OF SPECTRA AND INFINITE LOOP SPACES
}

\author{
by
}

\section{J. Peter May}

At the Seattle conference, I presented a calculation of $H_{*}\left(F ; Z_{p}\right)$ as an algebra, for odd primes $p_{\text {, }}$ where $F=\lim F(n)$ and $F(n)$ is the topological monoid of homotopy equivalences of an n-sphere. This computation was meant as a preliminary step towards the computation of $H^{*}\left(B F ; Z_{p}\right)$. Since then, I have calculated $H^{*}\left(B F ; z_{p}\right)$, for all primes $p$, as a Hopf algebra over the steenrod and Dyer-Lashof algebras. The calculation, while not difficult, is somewhat lengthy, and I was not able to write up a coherent presentation in time for inclusion in these proceedings. The computation required a systematic study of homology operations on $n$-fold and infinite loop spaces. As a result of this study, I have also been able to compute $H_{*}\left(\Omega^{n} S^{n} X_{i} Z_{p}\right)$, as a Hopf algebra over the steenrod algebra, for all connected spaces $\mathrm{X}$ and prime numbers $\mathrm{p}$. This result, which generalizes those of Dyer and Lashof [3] and Milgram [8], yields explicit descriptions of both $H_{*}\left(\Omega^{n} S^{n} X ; Z_{p}\right)$ and $H_{*}\left(Q X ; Z_{p}\right), Q X=l i m \Omega^{n} S^{n} X$, as functors of $H_{*}\left(x ; Z_{p}\right)$.

An essential first step towards these results was a systematic categorical analysis of the notions of $n$-fold and infinite loop spaces. The results of this analysis will 
be presented here. These include certain adjoint functor relationships that provide the conceptual reason that $\mathrm{H}_{\star}\left(\Omega^{\mathrm{n}} \mathrm{S}^{\mathrm{n}} \mathrm{X} ; \mathrm{Z}_{\mathrm{p}}\right)$ and $\mathrm{H}_{*}\left(Q \mathrm{X} ; \mathrm{Z}_{\mathrm{p}}\right)$ are functors of $\mathrm{H}_{*}\left(\mathrm{X} ; \mathrm{Z}_{\mathrm{p}}\right)$ and that precisely relate maps between spaces to maps between spectra. These categorical considerations motivate the introduction of certain non-standard categories, $I$ and $L$, of (bounded) spectra and $\Omega$-spectra, and the main purpose of this paper is to propagandize these categories. It is clear from their definitions that these categories are considerably easier to work with topologically than are the usual ones, but it is not clear that they are sufficiently large to be of interest. We shall remedy this by showing that, in a sense to be made precise, these categories are equivalent for the purposes of homotopy theory to the standard categories of (bounded) spectra and $\Omega$-spectra. We extend the theory to unbounded spectra in the last section.

The material here is quite simple, both as category theory and as topology, but it turns out nevertheless to have useful concrete applications. We shall indicate two of these at the end of the paper. In the first, we observe that there is a natural epimorphism, realized by a map of spaces, from the stable homotopy groups of an infinite loop space to its ordinary homotopy groups. In the second, by coupling our results with other information, we shall construct a collection of interesting topological spaces and 
maps; the other information by itself gives no hint of the possibility of performing this construction.

\section{THE CATEGORIES Ln AND HOMOLOGY}

In order to sensibly study the homology of iterated loop spaces, it is necessary to have a precise categorical framework in which to work. It is the purpose of this section to present such a framework.

We let $T$ denote the category of topological spaces with base-point and base-point preserving maps, and we let

$$
\mu: \operatorname{Hom}_{T}(\mathrm{X}, \Omega \mathrm{Y}) \longrightarrow \operatorname{Hom}_{\mathcal{T}}(\mathrm{SX}, \mathrm{Y})
$$

denote the standard adjunction homeomorphism relating the loop and suspension functors.

We define the category of $n$-fold loop sequences, $L_{n}$, to have objects $B=\left\{B_{i} \mid 0 \leq i \leq n\right\}$ such that $B_{i}=\Omega B_{i+1} \in T$ and maps $g=\left\{g_{i} \mid 0 \leq i \leq n\right\}$ such that $g_{i}=\Omega g_{i+1} \in T ;$ clearly $B_{0}=\Omega^{i} B_{i}$ and $g_{0}=\Omega^{i} g_{i}$ for $0 \leq i \leq n$. We define $L=L_{\infty}$ to be the category with objects $B=\left\{B_{i} \mid i \geq 0\right\}$ such that $B_{i}=\Omega B_{i+1} \in T$ and maps $g=\left\{g_{i} \mid i \geq 0\right\}$ such that $g_{i}=\Omega g_{i+1} \in T_{i}$ clearly $\mathrm{B}_{0}=\Omega^{i} \mathrm{~B}_{i}$ and $g_{0}=\Omega^{i} g_{i}$ for all $i \geq 0$. We call $L_{\infty}$ the category of perfect $\Omega$-spectra (or of infinite loop sequences). For all $n$, we define forgetful functors $U_{n}: L_{n} \rightarrow T$ by $U_{n} B=B_{0}$ and $U_{n} g=g_{0}$. of course, if $n<\infty, \quad U_{n} B$ and $U_{n} g$ are $n$-fold loop spaces and maps. We 
say that a space $x \in T$ is a perfect infinite loop space if $\mathrm{X}=\mathrm{U}_{\infty} \mathrm{B}$ for some object $\mathrm{B} \in L_{\infty}$ and we say that a map $f \in T$ is a perfect infinite loop map if $f=U_{\infty} g$ for some map $g \in L_{\infty}$.

We seek adjoints $Q_{n}: T \longrightarrow L_{n}$, I $\leq n \leq \infty$, to the functors $U_{n}$. For $n<\infty$, define $Q_{n} X=\left\{\Omega^{n-i} S^{n} X \mid 0 \leq i \leq n\right\}$ and $Q_{n} f=\left\{\Omega^{n-i} S^{n} f \mid 0 \leq i \leq n\right\}$. Clearly, $Q_{n} X$ and $Q_{n} f$ are objects and maps in $L_{n}$. For the case $n=\infty$, we first define a functor $Q: T \longrightarrow T$ by letting $\mathrm{QX}=\underset{\lim }{\longrightarrow} \Omega^{\mathrm{n}} \mathrm{S}^{\mathrm{n}} \mathrm{X}$, where the limit is taken with respect to the inclusions

$$
\Omega^{n} \mu^{-1}\left(I_{S^{n+1} X}\right): \Omega^{n} S^{n} X \rightarrow \Omega^{n+1} S^{n+1} X
$$

For $\mathrm{f}: \mathrm{X} \longrightarrow \mathrm{Y}$, we define $Q \mathrm{f}=\underline{\lim } \Omega^{\mathrm{n}} \mathrm{S}^{\mathrm{n}} \mathrm{f}: Q \mathrm{XX} \longrightarrow Q \mathrm{Y}$. It is clear that $Q X=\Omega Q S X$ and $Q f=\Omega Q S f$. We can therefore define a functor $Q_{\infty}: T \longrightarrow L_{\infty}$ by $Q_{\infty} X=\left\{\operatorname{QS}^{i} X \mid i \geq 0\right\}$ and $Q_{\infty} f=\left\{Q S^{i} f \mid i \geq 0\right\}$.

\section{Proposition 1}

For each $n, l \leq n \leq \infty$, there is an adjunction

$$
\phi_{n}: \operatorname{Hom}_{T}\left(X, U_{n} B\right) \rightarrow \operatorname{Hom}_{L_{n}}\left(Q_{n} X, B\right) .
$$

Proof. Observe first that the following two composites are the identity.

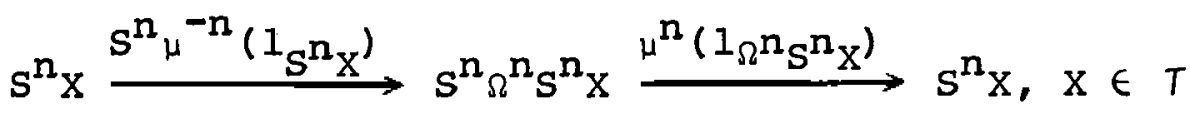

$$
\begin{aligned}
& \Omega^{n_{X}} \stackrel{\mu^{-n}\left(1_{S^{n}} n_{\Omega} n_{X}\right)}{\longrightarrow} \Omega^{n_{S}} S^{n} \Omega^{n} \stackrel{\Omega^{n}{ }^{n}\left(1_{\Omega} n_{X}\right)}{\longrightarrow} \Omega^{n_{X}}, x \in T
\end{aligned}
$$


In fact, since $\mu(f)=\mu\left(I_{\Omega Z}\right)$. SE for any map $\mathrm{E}: \mathrm{Y} \rightarrow \Omega \mathrm{Z}$ in $T, \mu^{n}\left(1_{\Omega} n_{S} n_{X}\right) \cdot S^{n_{\mu}-n}\left(l_{S} n_{X}\right)=\mu^{n_{\mu}-n}\left(1_{S} n_{X}\right)=1_{S} n_{X}$; this proves (1.2) and the proof of (1.3) is similar. Now define natural transformations $\Phi_{n}: Q_{n} U_{n} \rightarrow I_{L_{n}}$ and $\Psi_{n}: 1_{T} \longrightarrow U_{n} \Omega_{n}$ by

$$
\begin{aligned}
& \Phi_{n}(B)=\left\{\Omega^{n-i}{ }_{\mu}^{n}\left(I_{B_{0}}\right) \mid 0 \leq i \leq n\right\}: Q_{n} U_{n} B \rightarrow B \text { if } n<\infty \text {; } \\
& \Phi_{\infty}(B)=\left[\underline{\lim } \Omega^{j} \mu^{i+j}\left(1_{B_{0}}\right) \mid i \geq 0\right\}: Q_{\infty} U_{\infty} B \longrightarrow B \text { if } n=\infty ; \\
& \Psi_{n}(x)=\mu^{-n}\left(1_{S} n_{X}\right): x \rightarrow U_{n} Q_{n} x=\Omega^{n} S^{n} x \text { if } n<\infty ; \\
& \Psi_{\infty}(x)=\stackrel{\lim }{\longrightarrow} \mu^{-j}\left(1_{S} j_{X}\right): x \longrightarrow U_{\infty} Q_{\infty} x=Q X \text { if } n=\infty \text {. }
\end{aligned}
$$

We claim that $(1.2)$ and $(1.3)$,imply that the following two composites are the identity for all $n$.

$$
\begin{aligned}
& Q_{n} x \stackrel{Q_{n} \Psi_{n}(X)}{\longrightarrow} Q_{n} U_{n} Q_{n} X \stackrel{\Phi_{n}\left(Q_{n} X\right)}{\longrightarrow} Q_{n} X, x \in T \\
& U_{n} B \stackrel{\Psi_{n}\left(U_{n} B\right)}{\longrightarrow} U_{n} Q_{n} U_{n} B \stackrel{U_{n} \Phi_{n}(B)}{\longrightarrow} U_{n} B, B \in L_{n}
\end{aligned}
$$

For $n<\infty,(1.6)$ follows from $(1.2)$ by application of $\Omega^{\mathrm{n}-\mathrm{i}}$ for $0 \leq i \leq n$ and (1.7) is just (1.3) applied to $x=B_{n}$, since $B_{0}=U_{n} B=\Omega^{n} B_{n}$. For $n=\infty$, observe that $\Psi_{\infty}(x)$ factors as the composite

$$
\mathrm{X} \stackrel{\mu^{-1}\left(1_{S X}\right)}{\longrightarrow} \Omega S X \stackrel{\Omega \Psi_{\infty}(S X)}{\longrightarrow} \Omega Q S X=Q X .
$$

It follows that $\Psi_{\infty}(X)=\mu^{-i_{\Psi_{\infty}}\left(S^{i} X\right)}$ for all $i \geq 0$ since

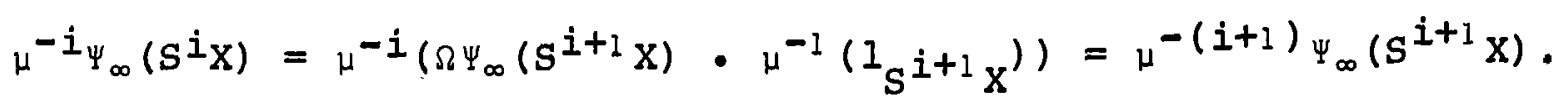
Observe also that

$$
\Omega_{\Omega}{ }_{\Psi_{\infty}}\left(S^{i+j} X\right): \Omega^{j} S^{i+j} X \longrightarrow \Omega^{j} Q S^{i+j} X=Q S^{i} X
$$


is just the natural inclusion obtained from the definition of $Q S^{i} x$ as $\stackrel{\lim }{\rightarrow} \Omega^{j} S^{i+j} x$. We therefore have that:

$$
\begin{aligned}
& \Phi_{\infty}\left(Q_{\infty} X\right)_{i} \cdot Q_{\infty} \Psi_{\infty}(X)_{i}
\end{aligned}
$$

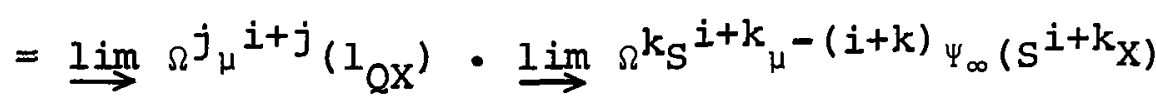

$$
\begin{aligned}
& =\lim _{\Omega} j_{\mu}^{i+j}\left(1_{Q X}\right) \cdot \Omega^{j} S^{i+j} j_{\mu}-(i+j)_{\Psi_{\infty}}\left(S^{i+j_{X}}\right) \\
& =\stackrel{\lim }{\longrightarrow} j_{\Psi_{\infty}}\left(S^{i+j_{X}}\right)=1_{Q S} i_{X}
\end{aligned}
$$$$
\begin{array}{r}
U_{\infty} \Phi_{\infty}(B) \cdot \Psi_{\infty}\left(U_{\infty} B\right)=\underset{\lim }{\longrightarrow} \Omega^{j} j^{j}\left(I_{B_{0}}\right) \cdot \stackrel{\lim }{\longrightarrow} \mu^{-k}\left(I_{S} k_{B_{0}}\right) \\
=\stackrel{\lim }{\longrightarrow} \Omega^{j}{ }^{j}\left(I_{B_{0}}\right) \cdot \mu^{-j}\left(I_{S} j_{B_{0}}\right)=\stackrel{\lim }{\longrightarrow} I_{B_{0}}=I_{B_{0}} .
\end{array}
$$

In both calculations, the second equality is an observation about the limit topology. The third equalities follow from formulas (1.2) and (1.3) respectively. Finally, define

$$
\begin{aligned}
& \phi_{n}(f)=\Phi_{n}(B) \cdot Q_{n} f \text { if } f: x \rightarrow U_{n} B \text { is a map in } T(1.8) \\
& \psi_{n}(g)=U_{n} g \cdot \psi_{n}(x) \text { if } g: Q_{n} x \rightarrow B \text { is a map in } L_{n} \text { (1.9) }
\end{aligned}
$$

It is a standard fact that $\phi_{\mathrm{n}}$ is an adjunction with inverse $\psi_{\mathrm{n}}$ since the composites $(1.6)$ and $(1.7)$ are each the identity. If $B \in L_{n}$, we define $H_{*}(B)=H_{*}\left(U_{n} B\right)$, where homology is taken with coefficients in any Abelian group $\pi$. We regard $H_{*}$ as a functor defined on $L_{n}$, but we deliberately do not specify a range category. Indeed, the problem of determining the homology operations on $n$-fold and (perfect) infinite loop spaces may be stated as that of obtaining an appropriate algebraic description of the range category. It 
follows easily from (1.2) and (1.5) of the proof above that $\Psi_{n}(X)_{*}: H_{*}(X) \longrightarrow H_{*}\left(U_{n} \ell_{n} X\right)$ is a monomorphism. since $Q_{n}$ is adjoint to $u_{n}$, the objects $\Omega_{n} x$ are, in a well-defined sense, free objects in the category $L_{n}$. It is therefore natural to expect $H_{*}\left(Q_{n} X\right)$ to be a functor of $H_{*}(X)$, with values in the appropriate range category. I have proven that this is the case if $\pi=z_{p}$ and have computed the functor. By the previous proposition, if $B \in L_{n}$ then any map $f: x \rightarrow U_{n} B$ in $T$ induces a map $\phi_{n}(f): Q_{n} X \rightarrow B$ in $L_{n}$, and the functor describing $H_{*}\left(Q_{n} X\right)$ is geometrically free in the sense that $\phi_{n}(f)_{*}: H_{*}\left(Q_{n} X\right) \rightarrow H_{*}(B)$ is determined by $f_{*}=U_{n} \phi_{n}(f)_{*} \Psi_{n}(X)_{*}: H_{*}(X) \longrightarrow H_{*}\left(U_{n} B\right)$ in terms of the homology operations that go into the definition of the functor. In this sense, we can geometrically realize enough free objects since $\Phi_{n}(B)_{*}: H_{*}\left(Q_{n} U_{n} B\right) \rightarrow H_{*}(B)$ is an epimorphism. All of these statements are analogs of wellknown facts about the cohomology of spaces. The category of unstable algebras over the steenrod algebra is the appropriate range category for cohomology with $\mathrm{z}_{\mathrm{p}}$-coefficients. Products of $K\left(z_{p}, n\right)$ 's play the role analogous to that of the $\Omega_{\mathrm{n}} \mathrm{X}$ and their fundamental classes play the role analogous to that of $H_{*}(X) \subset H_{*}\left(Q_{n} X\right)$.

By use of Proposition 1, we can show the applicability of the method of acyclic models to the homology of iterated loop spaces. The applications envisaged are to natural transformations defined for iterated loop spaces but 
not for arbitrary spaces. The argument needed is purely categorical. Let $T$ temporarily denote any category, let A denote the category of modules over a commutative ring $\Lambda$, and let $M$ be a set of model objects in $T$. Let $F: S \longrightarrow A$ be the free $\Lambda$-module functor, where $S$ is the category of sets. If $R: T \longrightarrow A$ is any functor, define a functor $\widetilde{R}: T \longrightarrow A$ by $\widetilde{R}(X)=F\left[\underset{M \in M}{\left.\operatorname{Hom}_{T}(M, X) \times R(M)\right]}\right.$ on objects and $\widetilde{R}(f)(\nu, r)=(f \cdot \nu, r)$ on morphisms, where if $f: X \longrightarrow Y$, then $\nu \in \operatorname{Hom}_{T}(M, X)$ and $r \in R(M)$. Define a natural transformation $\lambda: \widetilde{R} \rightarrow R$ by $\lambda(X)(v, r)=R(v)(r)$. Recall that $R$ is said to be representable by $M$ if there exists a natural transformation $\xi: R \rightarrow \widetilde{R}$ such that $\lambda \cdot \xi: R \rightarrow R$ is the identity natural transformation. With these notations, we have the following lemma.

Iemma $\underline{2}$

Let $\phi: \operatorname{Hom}_{T}(\mathrm{X}, \mathrm{UB}) \longrightarrow \mathrm{Hom}_{L}(\mathrm{QX}, \mathrm{B})$ be an adjunction and let $R: T \longrightarrow A$ be a functor representable by $M$. Define $Q M=\{Q M \mid M \in M\}$ and let $S=R \cdot U: L \longrightarrow A$. Then $S$ is representable by $Q M$.

Proof. Define a natural transformation $\eta: \widetilde{R} \cdot U \longrightarrow \widetilde{S}$ by $n(B)(\nu, r)=\left(\phi(v), R_{\phi}^{-1}\left(I_{Q M}\right)(r)\right)$ for $v: M \rightarrow U B, r \in R(M)$. Write $\lambda^{\prime}$ for the natural transformation $\mathbb{S} \longrightarrow S$ defined as above for $\widetilde{R}$. We have $\lambda^{\prime} n=\lambda \mathrm{U}: \widetilde{\mathrm{R} U} \longrightarrow \mathrm{RU}=\mathrm{S}$ since $\lambda^{\prime} n(B)(U, r)$ $=S \phi(v)\left[R \phi^{-1}\left(1_{Q M}\right)(r)\right]=R\left[U \phi(v) \cdot \phi^{-1}\left(1_{Q M}\right)\right](r)=R(v)(r)$. 
Therefore, if $\xi: R \longrightarrow \widetilde{R}$ satisfies $\lambda \xi=1: R \longrightarrow R$, then $\lambda^{\prime}(n \xi U)=\lambda U \cdot \xi U=1: S \rightarrow S$, and this proves the result. of course, if $\phi$ is an adjunction as in the lemma and if $T^{j}$ denotes the product of $j$ factors $T$, then $\phi^{j}: \operatorname{Hom}_{T} j\left(X, U^{j} B\right) \longrightarrow \operatorname{Hom}_{L}{ }^{j}\left(Q^{j} X, B\right)$ is also an adjunction $\left(X \in T^{j}, B \in L^{j}\right)$. Thus the lemma applies to functors $R: T^{j} \longrightarrow A$ and $R U^{j}: L^{j} \rightarrow A$. Returning to topology, let $c_{\star}: T \rightarrow A$ be the singular chain complex functor, with coefficients in $\Lambda$. The lemma applies to $C_{*} U_{n}: L_{n} \rightarrow A$ for $1 \leq n \leq \infty$ and, by the remark above, to the usual related functors on $L_{\mathfrak{n}}^{j}$ (tensor and Cartesian products of singular chain complexes). With $M=\left\{\Delta_{\mathfrak{m}}\right\}$, the standard set of models in $T$, we have $\mathrm{U}_{\mathrm{n}} \mathrm{Q}_{\mathrm{n}} \Delta_{\mathrm{m}}=\Omega^{\mathrm{n}} \mathrm{S}^{\mathrm{n}} \Delta_{\mathrm{m}}$ if $\mathrm{n}<\infty$ and $\mathrm{U}_{\infty} Q_{\infty} \Delta_{\mathrm{m}}=\mathrm{Q} \Delta_{\mathfrak{m}}$; these spaces are contractible and the model objects $\left\{Q_{\mathfrak{n}} \Delta_{\mathfrak{m}}\right\} \subset L_{n}$ are therefore acyclic. We conclude that the method of acyclic models [4] is applicable to the study of the homology of $\mathrm{n}$-fold and perfect infinite loop spaces.

\section{$\underline{2}$ COMPARISONS OF CATEGORIES OF SPECTRA}

The work of the previous section shows that the category $L$ is a reasonable object of study conceptually, but it is not obvious that $L$ is large enough to be of topological interest. For example, it is not clear that the infinite classical groups are homotopy equivalent to perfect infinite loop spaces. We shall show that, from the point of view of 
homotopy theory, $L$ is in fact equivalent to the usual category of (bounded) $\Omega$-spectra. To do this, we shall have to proceed by stages through a sequence of successively more restrictive categories of spectra.

By a spectrum, we shall mean a sequence $B=\left\{B_{i}, f_{i} \mid i \geq 0\right\}$, where $B_{i}$ is a space and $f_{i}: B_{i} \longrightarrow \Omega B_{i+1}$ is a map. By a map $g: B \longrightarrow B^{\prime}$ of spectra we shall mean a sequence of maps $g_{i}: B_{i} \longrightarrow B_{i}^{\prime}$ such that the following diagrams are homotopy commutative, i $\geq 0$.

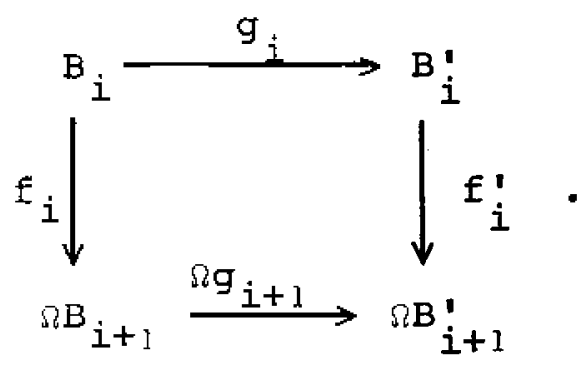

We call the resulting category $S$. We say that $B \in S$ is an inclusion spectrum if each $f_{i}$ is an inclusion. We obtain the category $I$ of inclusion spectra by letting a map in $I$ be a map in $S$ such that the diagrams (2.1) actually commute on the nose for each $i \geq 0$. (Thus, I is not a full subcategory of $\left.S_{.}\right)$We say that $B \in S$ is an $\Omega$-spectrum if each $f_{i}$ is a homotopy equivalence. We let $\Omega S$ be the full subcategory of $S$ whose objects are the $\Omega$-spectra, and we let $\Omega I=I \cap \Omega S$ be the full subcategory of $I$ whose objects are the inclusion $\Omega$-spectra. A spectrum $B \in \Omega I$ will be said to be a retraction spectrum if $B_{i}$ is a deformation retract of $\Omega B_{i+1}$ for all $i$. We let $R$ denote the 
full subcategory of $\Omega I$ whose objects are the retraction spectra. Clearly, $L$ is a full subcategory of $R$, since if $B \in L$ we may take $f_{i}=1$ and then any map in $R$ between objects of $L$ will be a map in $L$ by the commutativity of the diagrams (2.1). Thus we have the following categories and inclusions

$$
L \subset R \subset \Omega I \subset \Omega S \text { and } I \subset S \text {. }
$$

For each of these categories $C$, if $g^{\prime} g^{\prime}: B \longrightarrow B^{\prime}$ are maps in $\mathcal{C}$, then we say that $g$ is homotopic to $g^{\prime}$ if $g_{i}$ is homotopic to $g_{i}^{\prime}$ in $T$ for each $i$. We say that $g$ is a (weak) homotopy equivalence if each $g_{i}$ is a (weak) homotopy equivalence. Now each $\mathcal{C}$ has a homotopy category $\mathrm{HC}$ and a quotient functor $\mathrm{H}: \mathrm{C} \longrightarrow \mathrm{HC}$. The objects of $\mathrm{HC}$ are the same as those of $C$ and the maps of HC are homotopy equivalence classes of maps in $C$. Note that each of the inclusions of $(2.2)$ is homotopy preserving in the sense that if $C \subset D$ and $g \sim g^{\prime}$ in $C$, then $g \sim g^{\prime}$ in $D$. We therefore have induced functors $\mathrm{HC} \longrightarrow \mathrm{HD}$ and these are still inclusions since if $g, g^{\prime} \in C$ and $g \sim g^{\prime}$ in $D$, then $g \sim g^{\prime}$ in c.

The following definitions, due to Swan [11], will be needed in order to obtain precise comparisons of our various categories of spectra.

$\underline{\text { Definitions }} \underline{3}$

(i) A category $C$ is an H-category if there is an 
equivalence relation $\sim$, called homotopy, on its hom sets such that $f \sim f^{\prime}$ and $g \sim g^{\prime}$ implies $f g \sim f^{\prime} g^{\prime}$ whenever $\mathrm{fg}$ is defined. We then have a quotient category $\mathrm{HC}$ and a quotient functor $\mathrm{H}: \mathrm{C} \longrightarrow \mathrm{HC}$.

(ii) Let $C$ be any category and $D$ an H-category. A prefunctor $T: C \longrightarrow D$ is a function, on objects and maps, such that HT: $\mathrm{C} \longrightarrow \mathrm{HD}$ is a functor. This amounts to requiring $T\left(I_{C}\right) \sim I_{T(C)}$ for each $C \in C$ and $T(f g) \sim T(f) T(g)$ whenever $\mathrm{fg}$ is defined in $\mathrm{C}$. If $\mathrm{C}$ is also an H-category, we say that a prefunctor $T: C \longrightarrow D$ is homotopy preserving if $f \sim g$ in $C$ implies $T(f) \sim T(g)$ in 0 . Clearly, $T$ is homotopy preserving if and only if $T$ determines a functor $\mathrm{T}_{*}: \mathrm{HC} \longrightarrow \mathrm{HD}$ such that $\mathrm{HT}=\mathrm{T}_{*} \mathrm{H}$.

(iii) Let $S, T: C \longrightarrow D$ be prefunctors. A natural transformation of prefunctors $n: S \longrightarrow T$ is a collection of maps $n(C): S(C) \longrightarrow T(C), C \in C$, such that $T(f) \eta(C) \sim n\left(C^{\prime}\right) S(f)$ in $D$ for each map $f: C \longrightarrow C^{\prime}$ in $C$. $n$ is said to be a natural equivalence of prefunctors if there exists a natural transformation of prefunctors $\xi: T \longrightarrow S$ such that $n(C) \xi(C) \sim 1_{T(C)}$ and $\xi(C) n(C)=1_{S(C)}$ for each $c \in C$. A natural transformation of prefunctors $n: S \longrightarrow T$ determines a natural transformation of functors $\mathrm{Hn}: \mathrm{HS} \longrightarrow \mathrm{HT}$ and, if $S$ and $T$ are homotopy preserving, a natural transformation of functors $n_{*}: S_{*} \longrightarrow T_{*}$ such that $n_{*} H=H \eta$ if $n$ is a natural equivalence of prefunctors, then $H n$ and, if defined, $n_{*}$ are natural equivalences of functors. 
(iv) If $S: D \longrightarrow C$ and $T: C \longrightarrow D$ are homotopy preserving prefunctors between H-categories, we say that $T$ is adjoint to $\mathrm{s}$ if there exist natural transformations of prefunctors $\Phi:$ TS $\longrightarrow 1_{D}$ and $\Psi: 1_{C} \rightarrow$ ST such that for each $D \in D$ the composite $S \Phi(D) \Psi(S D): S D \rightarrow S D$ is homotopic in $C$ to the identity map of $S D$ and for each $C \in \mathcal{C}$ the composite $\Phi(T C) \cdot T \Psi(C): T C \longrightarrow T C$ is homotopic in $D$ to the identity map of TC. If $S$ and $T$ are adjoint prefunctors, then $\mathrm{S}_{*}: \mathrm{HD} \longrightarrow \mathrm{HC}$ and $\mathrm{T}_{*}: \mathrm{HD} \longrightarrow \mathrm{HC}$ are adjoint functors, with adjunction $\phi_{*}=\Phi_{*} \mathrm{~T}_{*}: \operatorname{Hom}_{\mathrm{HC}}\left(\mathrm{A}, \mathrm{S}_{*} \mathrm{~B}\right) \longrightarrow \mathrm{Hom}_{\mathrm{HD}}\left(\mathrm{T}_{*} \mathrm{~A}, \mathrm{~B}\right)$. We can now compare our various categories of spectra. The following theorem implies that $I$ is equivalent to $S$ for the purposes of homotopy theory in the sense that no homotopy invariant information is lost by restricting attention to spectra and maps of spectra in $I$, and that $\Omega I$ is equivalent to $\Omega S$ in this sense. Under restrictions on the types of spaces considered, it similarly compares $R$ to $\Omega S$. To state the restrictions, let $C$ denote the full subcategory of $S$ whose objects are those spectra $\left\{B_{i}, f_{i}\right\}$ such that each $B_{i}$ is a locally finite countable simplicial complex and each $\mu\left(f_{i}\right): S B_{i} \longrightarrow B_{i+1}$ is simplicial. Observe that if $w$ is the full subcategory of $S$ whose objects are those spectra $B$ such that each $B_{i}$ has the homotopy type of a countable $\mathrm{CW}$-complex, then every object of $W$ is homotopy equivalent (in $S)$ to an object of $C$. In fact, if $\left\{B_{i}, f_{i}\right\} \in W$, then each $B_{i}$ is homotopy equivalent to a locally finite simplicial 
complex $B_{i}^{\prime}$ by $\left[9\right.$, Theorem 1]; if $f_{i}^{\prime}$ is the composite $\mathrm{B}_{i}^{\prime} \longrightarrow \mathrm{B}_{i} \stackrel{\mathrm{f}_{i}}{\longrightarrow} \Omega \mathrm{B}_{i+1} \longrightarrow \Omega \mathrm{B}_{i+1}^{\prime}$ determined by chosen homotopy equivalences $B_{i} \rightleftarrows B_{i}^{\prime}$ and if $\mu\left(f_{i}^{\prime \prime}\right)$ is a simplicial approximation to $\mu\left(f_{i}^{\prime}\right)$, then $\left\{B_{i}, f_{i}\right\}$ is homotopy equivalent to $\left\{B_{i}^{\prime}, f_{i}^{\prime}\right\}$ and therefore to $\left\{B_{i}^{\prime}, f_{i}^{\prime}\right\} \in C$.

Theorem $\underline{4}$

There is a homotopy preserving prefunctor $M: S \longrightarrow I$ such that

(i) There exists a natural equivalence of prefunctors $\eta: 1_{S} \longrightarrow J M$, with inverse $\xi: J M \longrightarrow 1_{S}$, where $\mathrm{J}: I \longrightarrow S$ is the inclusion. Therefore $\mathrm{J}_{*} \mathrm{M}_{*}$ is naturally equivalent to the identity functor of HS.

(ii) MJ: $I \longrightarrow I$ is a functor, $\xi(J B): J M J B \longrightarrow J B$ is a map in $I$ if $B \in I$, and if $\zeta: M J \longrightarrow I_{I}$ is defined by $\zeta(B)=\xi(J B)$, then $\zeta$ is a natural transformation of functors.

(iii) $\eta$ and $\zeta$ establish an adjoint prefunctor relationship between $J$ and $M$. Therefore $\phi_{*}: \operatorname{Hom}_{H S}\left(A, J_{*} B\right) \longrightarrow \operatorname{Hom}_{H I}\left(M_{*} A, B\right)$ is an adjunction, where $\phi_{*}(f)=\zeta_{*}(B) M_{*} f, f: A \longrightarrow J_{*} B$, and $\phi_{*}^{-1}(g)=J_{*} g \cdot \eta_{*}(A)$, g: $M_{\star} A \longrightarrow B$.

(iv) By restriction, $M$ induces a homotopy preserving prefunctor $\Omega S \longrightarrow \Omega I$ which satisfies (i) through (iii) with respect to the inclusion $\Omega I \longrightarrow \Omega S$.

(v) By restriction, $M$ induces a homotopy 
preserving prefunctor $\Omega S \cap C \longrightarrow R \cap C$ which satisfies (i) through (iii) with respect to the inclusion $R \cap \mathcal{C} \rightarrow \Omega S \cap C$.

Proof. We first construct $M$ and prove (i) and

(ii) simultaneously. Let $B=\left\{B_{i}, f_{i}\right\} \in S$. Define $M B=\left\{M_{i} B, M_{i} f\right\} \in I$ by induction on $i$ as follows. Let $M_{0} B=B_{0}$. Assume that $M_{j} B, j \leq i$, and $M_{j} E_{1} j<i$, have been constructed. Let $n_{0}=1=\xi_{0}$ and assume further that $n_{j}: B_{j} \longrightarrow M_{j} B$ and $\xi_{j}: M_{j} B \longrightarrow B_{j}$ have been constructed such that

(a) $\xi_{j} \eta_{j}=1: B_{j} \longrightarrow B_{j}$ and $\eta_{j} \xi_{j} \sim 1: M_{j} B \longrightarrow M_{j} B$; (b) $\quad \Omega \xi_{j} \cdot M_{j-1} f=f_{j-1} \cdot \xi_{j-1}$ and $\Omega \eta_{j} \cdot f_{j-1}=M_{j-1} f \cdot \eta_{j-1} \cdot$ Define $M_{i+1} B$ to be the mapping cylinder of the map $\mu\left(f_{i}\right) \cdot S \xi_{i}: S M_{i} B \longrightarrow B_{i+1}$, let $k_{i}: S M_{i} B \longrightarrow M_{i+1} B$ denote the standard inclusion, and define $M_{i} f=\mu^{-1}\left(k_{i}\right): M_{i} B \longrightarrow \Omega M_{i+1} B$. Clearly $M_{i} f$ is then an inclusion. Consider the diagram

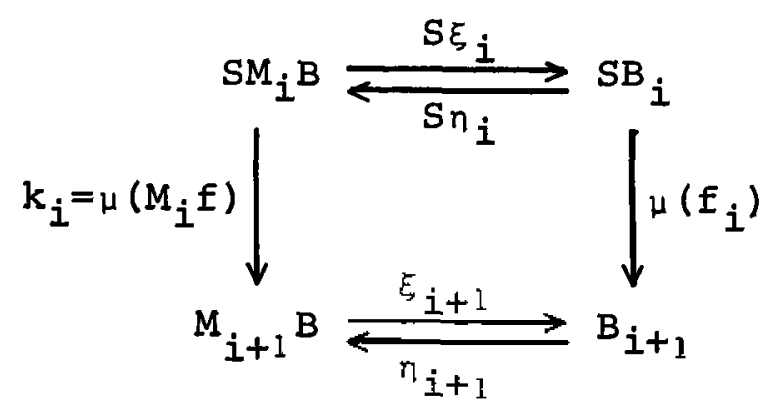

Here $\eta_{i+1}$ and $\xi_{i+1}$ are the inclusion and retraction obtained by the standard properties of mapping cylinders, hence (a) is satisfied for $j=i+1$. It is standard that $\xi_{i+1} \cdot \mu\left(M_{i} f\right)=\mu\left(f_{i}\right) S \xi_{i}$, and $\Omega \xi_{i+1} \cdot M_{i} f=f_{i} \xi_{i}$ follows by 
application of $\mu^{-1}$. Now $\Omega \eta_{i+1} \cdot f_{i} \sim M_{i} E \cdot \eta_{i}$ is obtained by a simple chase of the diagram. This proves (b) for $j=i+1$ and thus constructs $M$ on objects and constructs maps $n(B): B \longrightarrow J M B$ and $\xi(B): J M B \longrightarrow B$ in $S$. If $B \in I$, then $\xi(J B)$ is a map in $I$ by (b) and we can define $\zeta(B)=\xi(J B): M J B \longrightarrow B$. We next construct $M$ on maps. Let $g: B \longrightarrow B^{\prime}$ be a map in $S$. Define $M_{0} g=g_{0}$ and assume that $M_{j} g$ have been found for $j \leq i$ such that (with

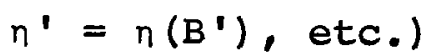

(c) $\eta_{j}^{\prime} g_{j}=M_{j} g \cdot \eta_{j} ; \xi_{j}^{\prime} \cdot M_{j} g \sim g_{j} \xi_{j}$ with equality if $g \in I$; (d) $\Omega M_{j} g \cdot M_{j-1} f=M_{j-1} f^{\prime} \cdot M_{j-1} g \cdot$ Then, by (c) and the definition of maps in the categories $S$ and $I, f_{i}^{\prime} \xi_{i}^{\prime} M_{i} g \sim f_{i}^{\prime} g_{i} \xi_{i} \sim \Omega g_{i+1} f_{i} \xi_{i}: M_{i} B \longrightarrow \Omega B_{i+1}$, with equalities if $g \in I$. Applying $\mu$, we see that there exists a homotopy $h_{i}: S_{i} B \times I \rightarrow B_{i+1}^{\prime}$ from $\mu\left(f_{i}^{\prime}\right) S \xi_{i}^{\prime} S_{i} g$ to $g_{i+1} \mu\left(f_{i}\right) S \xi_{i}$, and we agree to choose $h_{i}$ to be the constant homotopy if $g \in I$. Write $[x, t]$ and $[y]$ for the images of $(x, t) \in S_{i} B \times I$ and $y \in B_{i+1}$ in the mapping cylinder $M_{i+{ }_{1}}{ }$ of $\mu\left(f_{i}\right) S \xi_{i}$, and similarly for $M_{i+1} B^{\prime}$. Define $M_{i+1} g: M_{i+1} B \longrightarrow M_{i+1} B^{\prime}$ by

(e) $M_{i+1} g[x, t]= \begin{cases}{\left[\mathrm{SM}_{i} g(x), 2 t\right],} & 0 \leq t \leq 1 / 2 \\ {\left[\mathrm{~h}_{i}(x, 2 t-1)\right],} & 1 / 2 \leq t \leq 1 .\end{cases}$

$$
M_{i+1} g[y]=\left[g_{i+1}(y)\right] \text {. }
$$

It is trivial to verify that $M_{i+1} g$ is well-defined and continuous. Now consider the following diagram: 


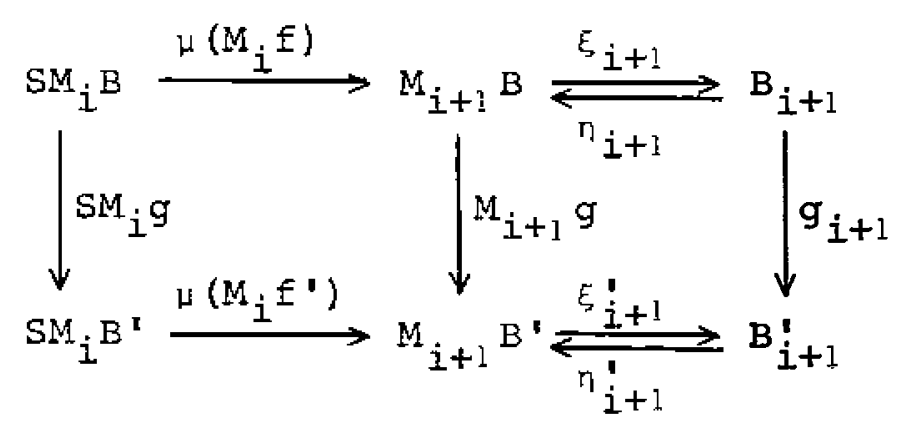

Since $n_{i+1}(y)=[y], n_{i+1}^{\prime} \cdot g_{i+1}=M_{i+1} g \cdot n_{i+1}$ is obvious, and $\xi_{i+1}^{i} \cdot M_{i+1} g \sim g_{i+1} \xi_{i+1}$ then follows from (a) and a simple chase of the right-hand square. If the map $g$ is in I, then $\xi_{i+1}^{\prime} M_{i+1} g=g_{i+1} \xi_{i+1}$ is easily verified by explicit computation since $h_{i}(x, t)=g_{i+1} \mu\left(f_{i}\right) S \xi_{i}(x)$ for all $t$. This proves (c) for $j=i+1$. To prove (d) for $j=i+1$, merely observe that the left-hand square clearly commutes, since $\mu\left(M_{i} f\right)(x)=[x, 0]$, and apply $\mu^{-1}$ to this square. of course, (d) proves that $\mathrm{M}_{\mathrm{g}}$ is a map in $I$, and (c) completes the proof of (ii) of the theorem since $\mathrm{MJ}: I \longrightarrow I$ is clearly a functor. If $\ell: M_{i+1} \mathrm{~B}^{\longrightarrow} \mathrm{M}_{i+1} \mathrm{~B}^{\prime}$ is any map whatever such that $l n_{i+1} \sim n_{i+1}^{\prime} g_{i+1}$, then

$$
M_{i+1} g \simeq n_{i+1}^{\prime} \xi_{i+1}^{\prime} M_{i+1} g \simeq n_{1+1}^{\prime} g_{i+1} \xi_{i+1} \simeq \ln _{i+1} \xi_{i+1} \approx \ell \text {. }
$$

It follows that the homotopy class of $M_{i+1} g$ is independent of the choice of $h_{i}$, and from this it follows easily that $M: S \longrightarrow I$ is a prefunctor. $M$ is homotopy preserving since if $g \sim g^{\prime}: B \longrightarrow B^{\prime}$ in $S$, then $M_{i} g \sim M_{i} g \cdot n_{i} \xi_{i}=n_{i}^{\prime} g_{i} \xi_{i}=n_{i}^{\prime} g_{i}^{\prime} \xi_{i}=M_{i} g^{\prime} \cdot n_{i} \xi_{i}=M_{i} g^{\prime}$, $i \geq 0$. Now (i) of the theorem follows immediately from (a), (b), and (c).

(iii) To prove (iii), we must show that the following 
two composites are homotopic to the identity map.

(f) $\mathrm{JB} \stackrel{\eta(J B)}{\longrightarrow} \mathrm{JMJB} \stackrel{\mathrm{J} \zeta(B)}{\longrightarrow} \mathrm{JB}, \mathrm{B} \in I$

(g) $\mathrm{MB} \stackrel{\mathrm{Mn}(\mathrm{B})}{\longrightarrow} \mathrm{MJMB} \stackrel{\zeta(\mathrm{MB})}{\longrightarrow} \mathrm{MB}, \mathrm{B} \in S$.

By (a) and $\zeta(B)=\xi(J B)$, the composite $(f)$ is the identity map. For $(g)$, note that $\xi(J M B) \eta(J M B)=1 \sim \eta(B) \xi(B): J M B \longrightarrow J M B$. By the uniqueness proof above for the homotopy class of $M_{i+1} g$ applied to the case $g=\xi(B)$, we have $M \xi(B) \sim \xi$ (JMB) $=\zeta(M B)$. Since $M \xi(B) M n(B) \sim 1$ by the fact that $M$ is a prefunctor, this proves that the composite $(g)$ is homotopic to the identity.

(iv) Since $\Omega S$ and $\Omega I$ are full subcategories of $S$ and $I$, it suffices for (iv) to prove that $M B \in \Omega I$ if $B \in \Omega S$, and this follows from (a) and (b) which show that if $g_{j}: \Omega B_{j+1} \longrightarrow B_{j}$ is a homotopy inverse to $f_{j}$, then $\eta_{j} g_{j} \Omega \xi_{j+1}: \Omega M_{j+1} B \longrightarrow M_{j} B$ is a homotopy inverse to $M_{j} f$. (v) Again, it suffices to show that $M B \in R \cap C$ if $B \in \Omega S \cap C$. By induction on $i$, starting with $M_{0} B=B$ and $n_{0}=1=\xi_{0}$, we see that each $M_{i} B$ is a locally finite countable simplicial complex and that each map $\mu\left(M_{i-1} f\right), \eta_{i}$ and $\xi_{i}$ is simplicial, since $\mathrm{M}_{i+{ }_{1}} \mathrm{~B}$ is the mapping cylinder of the simplicial map $\mu\left(f_{i}\right) S \xi_{i}: S M_{i} B \longrightarrow B_{i+1}[10, p .151]$. By Hanner [5, Corollary 3.5], every countable locally finite simplicial complex is an absolute neighborhood retract (ANR) and, by Kuratowski [7, p. 284], the loop space of an ANR is an ANR. Since the image of $M_{i} f$ is a closed subspace of the 
ANR $\Omega M_{i+1} B, M_{i} f$ has the homotopy extension property with respect to the ANR $M_{i} B \quad[6, p .86]$, and therefore $M_{i} B$ is a deformation retract of $\Omega M_{i+1} B \quad[10$, p. 31]. This proves that $M B \in R \cap C$, as was to be shown.

The category $I$ is not only large and convenient. It is also conceptually satisfactory in view of the following observation relating maps in $T$ to maps in $I$. We can define a functor $\Sigma: T \longrightarrow I$ by letting $\Sigma X$ be the suspension spectrum of $x, \Sigma_{i} X=S^{i} X$ and $f_{i}=\mu^{-1}\left(1_{S} i+1_{X}\right)$. If $g: X \rightarrow Y$ is a map in $T$, define $\Sigma_{i} g=S^{i} g$; it is clear that $\Sigma g$ is in fact a map in $I$. Let $U=U_{I}: I \longrightarrow T$ be the forgetful functor, $U B=B_{0}$ and $U g=g_{0}$. Observe that UL: $T \longrightarrow T$ is the identity functor. With these notations, we have the following proposition.

\section{Proposition $\underline{5}$}

$\mathrm{U}: \operatorname{Hom}_{I}(\Sigma \mathrm{X}, \mathrm{B}) \longrightarrow \mathrm{Hom}_{T}(\mathrm{X}, \mathrm{UB})$ is an adjunction. Proof. If $B=\left\{B_{i}, f_{i}\right\} \in I$, define $f^{i}: B_{0} \longrightarrow \Omega^{i} B_{i}$ inductively by $f^{0}=1, f^{l}=f_{0}$, and $f^{i+1}=\Omega^{i} f_{i} \cdot f^{i}$ if $i>0$. Define a natural transformation $\Phi: \Sigma U \longrightarrow 1_{I}$ by $\Phi(B)=\left\{\mu^{i}\left(f^{i}\right)\right\}: \Sigma U B \longrightarrow B$. Since $\Omega \mu^{i+1}\left(f^{i+1}\right) \cdot \mu^{-1}\left(1_{S^{i+1}} B_{0}\right)$ $=\mu^{i}\left(f^{i+1}\right)=\mu^{i}\left(\Omega^{i} f_{i} \cdot f^{i}\right)=f_{i} \mu^{i}\left(f^{i}\right), \Phi(B)$ is a map in $I$. For $g: \mathrm{X} \longrightarrow \mathrm{UB}$, define $\phi(g)=\Phi(B) \Sigma g$. Clearly $\quad \mathrm{U} \phi(g)=\mu^{0}\left(f^{0}\right) \Sigma_{0} g$ $=g$. Now $f^{i}$ for $\Sigma X$ is easily verified to be $\mu^{-i}\left(l_{S}{ }^{i}\right): X \rightarrow \Omega^{i} S^{i} X$. Therefore $\Phi(\Sigma X)=I: \Sigma X \longrightarrow \Sigma X ;$ since 
we obviously have $\Sigma U\left(1_{\Sigma X}\right)=1: \Sigma X \rightarrow \Sigma U \Sigma X=\Sigma X$, this implies that $\phi \mathrm{U}=1$.

Finally, we compare $L$ to the categories $I, \Omega I$, and $R$. The following theorem shows that $L$ is nicely related conceptually to $I$ and is equivalent for the purposes of weak homotopy theory to $\Omega I$ in the sense that no weak homotopy invariant information is lost by restricting attention to spectra and maps of spectra in $L$; coupled with the remarks preceding Theorem 4, it also shows that $L \cap W$ is equivalent to $R \cap W$ for the purposes of homotopy theory.

$\underline{\text { Theorem }} \underline{6}$

There is a functor $L: I \longrightarrow L$ and a natural transformation of functors $n: I_{I} \rightarrow K L$, where $K: I \longrightarrow I$ is the inclusion, such that

(i) LK: $L \longrightarrow L$ is the identity functor and

$L: \operatorname{Hom}_{I}(A, K B) \rightarrow \operatorname{Hom}_{L}(L A, B)$

is an adjunction with $L^{-1}(g)=\mathrm{Kg} \cdot n(A)$ for $g: L A \rightarrow B$.

(ii) If $g \sim g^{\prime}$ in $I$, then $L g$ is weakly homotopic to $\mathrm{Lg}^{\prime}$ in $L$, and if $B \in \Omega I$, then $\eta(B): B \longrightarrow K L B$ is a weak homotopy equivalence.

(iii) Let $B \in R \cap C$; then $\eta(B): B \rightarrow K L B$ is a homotopy equivalence and if $g \sim g^{\prime}: B \longrightarrow B^{\prime}$ in $I$, then $L g \simeq L^{\prime}: L B \longrightarrow L B^{\prime}$ in $L$.

Proof. Let $B=\left\{B_{i}, f_{i}\right\} \in I$. Since each $f_{i}$ is an inclusion, we can define $I_{i} B=\stackrel{\lim }{\rightarrow} \Omega^{i_{B}}{ }_{i+j}$, where the limit is 
taken with respect to the inclusions $\Omega j_{f_{i+j}}: \Omega_{B} j_{i+j} \longrightarrow \Omega^{j+1} B_{i+j+I}$. Clearly $\Omega L_{i+1} B^{\prime}=I_{i} B$, hence $L B \in L$. If $g: B \longrightarrow B^{\prime}$ is a map in $I$, define $L_{i} g=\underset{l i m}{\longrightarrow} \Omega_{j} j_{i+j}: L_{i} B \longrightarrow I_{i} B^{\prime}$; the limit makes sense since $\Omega_{\Omega} j_{f_{i+j}{ }^{j}} j_{g_{i+j}}=\Omega^{j+1} g_{i+j+1} \Omega_{f_{i+j}}$ by the definition of maps in $I$. Clearly $\Omega I_{i+1} g=I_{i} g$, hence $I g \in L$. Define $n: I_{I} \longrightarrow K L$ by letting $n_{i}(B): B_{i} \longrightarrow I_{i} B$ be the natural inclusion; $n(B)$ is obviously a map in $I$ since $\Omega \eta_{i+1}(B) \cdot f_{i}$ $=n_{i}(B)$. Now (ii) of the theorem is a standard consequence of the definition of the limit topology. The fact that LK is the identity functor of $L$ is evident, and $n K: K \longrightarrow K L K$ and Ln: I $\longrightarrow$ LKL are easily verified to be the identity natural transformations. This implies (i) and it remains to prove (iii). If $B \in R$, with retractions $r_{i}: \Omega B_{i+1} \longrightarrow B_{i}$, define maps $r^{i j}: \Omega j_{B_{i+j}} \longrightarrow B_{i}$ inductively by $r^{i 0}=1, r^{i l}=r_{i}$, and $r^{i, j+1}=r^{i j} j_{\Omega} r_{i+j}$ if $j>0$. since $r_{i+j} f_{i+j}=1$, we have

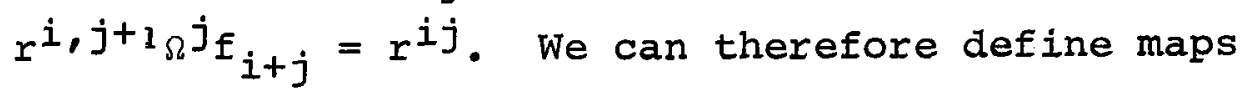
$\xi_{i}=\lim r^{i j}: I_{i} B \longrightarrow B_{i}$. Obviously $\xi_{i} n_{i}: B_{i} \longrightarrow B_{i}$ is the identity map. Suppose further that $B \in C$. Then we claim that $n_{i} \xi_{i} \sim I: L_{i} B \longrightarrow L_{i} B$. As in the proof of $(v)$ of Theorem 4 , each $\Omega j_{B_{i+j}}$ is now an ANR. Let us identify $\Omega j_{B_{i+j}}$ with its image under $\Omega^{j} f_{i+j}$ in $\Omega^{j+1} B_{i+j+1}$ for all $i$ and $j$ and omit the inclusion maps $\Omega^{j} f_{i+j}$ from the notation. Then the inclusion

$$
\Omega^{j} B_{i+j} \times I \cup \Omega^{j+1} B_{i+j+1} \times \dot{I} \subset \Omega^{j+1} B_{i+j+1} \times I
$$

is that of a closed subset in an ANR, and it therefore has the homotopy extension property with respect to the ANR $\Omega^{j+1} B_{i+j+1}$. 
In particular, by $[10$, p. 31$]$, each $B_{i}$ is a strong deformation retract of $\Omega B_{i+1}$, and we assume given homotopies $k_{i}: \Omega B_{i+1} \times I \longrightarrow \Omega B_{i+1}, k_{i}: 1 \sim r_{i}$ reI $B_{i}$. The $k_{i}$ induce homotopies: $k_{i j}: \Omega^{j+1} B_{i+j+1} \times I \longrightarrow \Omega^{j+1} B_{i+j+1}$, $k_{i j}: 1=\Omega^{j} r_{i+j}$ rel $\Omega j_{B_{i+j}}$, in the obvious fashion $\left(k_{i j, t}=\Omega j_{k_{i+j, t}}\right)$. We claim that, by induction on $j$, we can choose homotopies $h_{i j}: \Omega j_{B_{i+j}} \times I \longrightarrow \Omega j_{B_{i+j}}$, $h_{i j}: 1 \sim r^{i j}$ rel $B_{i}$, such that $h_{i, j+1}=h_{i j}$ on $\Omega j_{B_{i+j}} \times I$. To see this, let $h_{i_{0}}$ be the constant homotopy, let $h_{i l}=k_{i}=k_{i 0}$, and suppose given $h_{i j}$ for some $j>0$. Consider the following diagram:

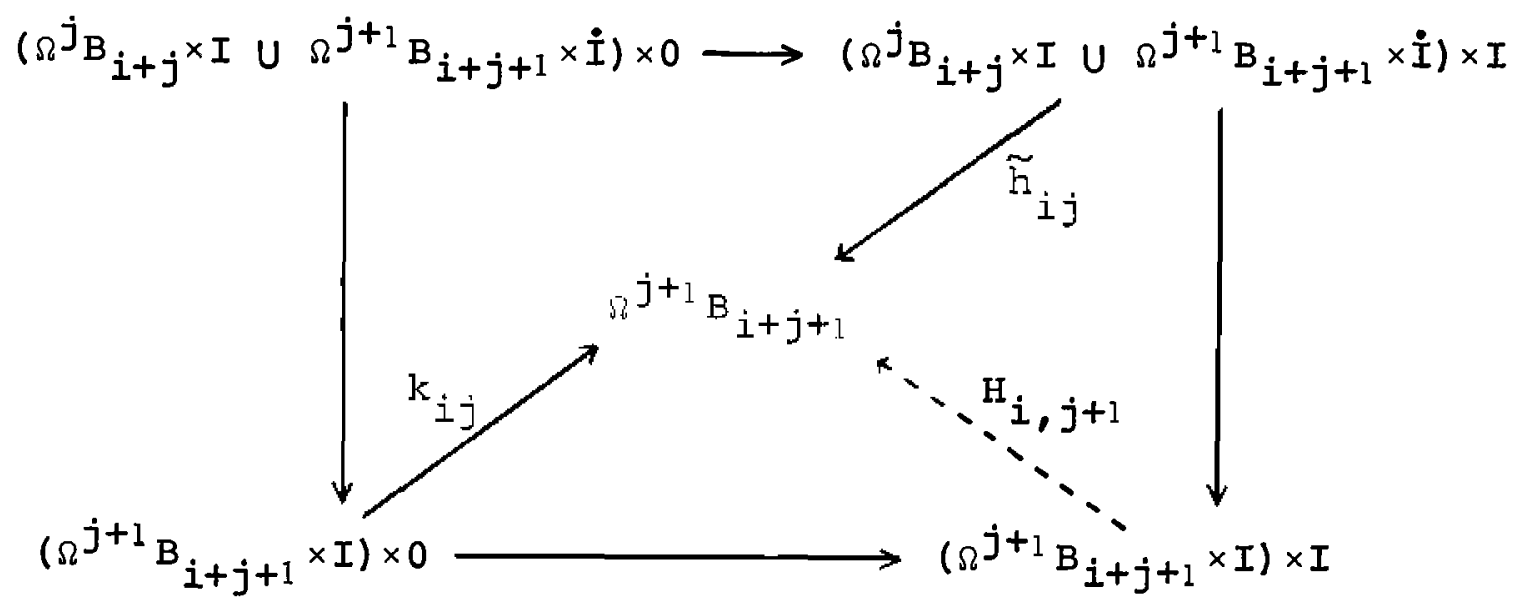

The unlabeled arrows are inclusions, and $\tilde{h}_{i j}$ is defined by $\tilde{h}_{i j}(x, s, t)=h_{i j}(x, s t)$ if $x \in \Omega j_{B_{i+j}} ;$ and $\tilde{h}_{i j}(y, 0, t)=y$, $\tilde{h}(y, I, t)=h_{i j}\left(\Omega^{j} r_{i+j}(y), t\right)$ if $y \in \Omega^{j+1} B_{i+j+1}$. It is easily verified that $\tilde{h}_{i j}$ is well-defined and continuous and that $\tilde{h}_{i j}=k_{i j}$ on the common parts of their domains. We can therefore obtain $\mathrm{H}_{i, j+1}$ such that the diagram commutes. Define $h_{i, j+1}(x, s)=H_{i, j+1}(x, s, 1)$. It is trivial to verify that 
$h_{i, j+1}$ has the desired properties. Now $\stackrel{\lim }{\longrightarrow} h_{i j}: I_{i} B \times I \longrightarrow I_{i} B$ is defined and is clearly a homotopy from 1 to $\eta_{i} \xi_{i}$. Finally, if $g \sim g^{\prime}: B^{\prime} \longrightarrow B^{\prime}$ in $I$ and $B \in R \cap \mathcal{C}$, then

$$
I_{i} g=I_{i} g \eta_{i} \xi_{i}=\eta_{i}^{\prime} g_{i} \xi_{i}=n^{\prime} g_{i}^{\prime} \xi_{i}=I_{i} g^{\prime} \eta_{i} \xi_{i} \sim I_{i} g^{\prime}, i \geq 0 .
$$

This completes the proof of (iii) and of the theorem.

We remark that the categorical relationships of

Propositions 1 and 5 and of the theorem are closely related. In fact, the composite functor $L \Sigma: T \longrightarrow L$ is precisely $Q_{\infty}$, and the adjunction

$$
\phi_{\infty}: \operatorname{Hom}_{T}\left(\mathrm{X}, \mathrm{U}_{\infty} \mathrm{B}\right) \longrightarrow \mathrm{Hom}_{L}\left(Q_{\infty} \mathrm{X}, \mathrm{B}\right)
$$

of Proposition 1 factors as the composite $\left(U_{\infty}=U\right)$

$$
\operatorname{Hom}_{T}(\mathrm{X}, \mathrm{UKB}) \stackrel{\mathrm{U}^{-1}}{\longrightarrow} \operatorname{Hom}_{I}(\Sigma \mathrm{X}, \mathrm{KB}) \stackrel{\mathrm{L}}{\longrightarrow} \operatorname{Hom}_{L}\left(Q_{\infty} \mathrm{X}, \mathrm{B}\right) \text {. }
$$

The verification of these statements requires only a glance at the definitions.

\section{INEINITE LOOP SPACES}

We shall here summarize the implications of the work of the previous section for infinite loop spaces and give the promised applications. We then make a few remarks about the extension of our results to unbounded spectra and point out an interesting collection of connective cohomology theories.

It is customary to say that $x \in T$ is an infinite loop space if $X$ is the initial space $B_{0}$ of an $\Omega$-spectrum $B$. If $X$ is given as an $H$-space, it is required that its product 
be homotopic to the product induced from the homotopy equivalence $x \longrightarrow \Omega B_{1}$. Similarly, a map $f \in T$ is said to be an infinite loop map if $f$ is the initial map $g_{0}$ of a map of $\Omega$-spectra $g$. The functor $M: \Omega S \longrightarrow \Omega I$ of $T$ heorem 4 satisfies $M_{0} B=B_{0}$ and $M_{0} g=g_{0}$. We therefore see that the identical infinite loop spaces and maps are obtained if we restrict attention to inclusion $\Omega$-spectra and maps in $I$. If $f: x \rightarrow x^{\prime}$ is any infinite loop map, then Theorem 6 implies the existence of a commutative diagram of infinite loop maps

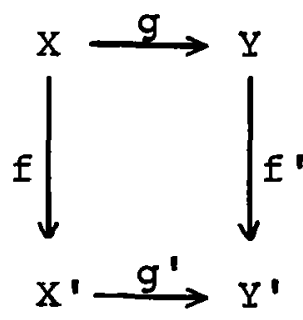

such that $f^{\prime}$ is a perfect infinite loop map between perfect infinite loop spaces and $g$ and $g^{\prime}$ are weak homotopy equivalences.

If $x$ is an infinite loop space of the homotopy type of a countable $\mathrm{CW}$-complex, then it follows from arguments of Boardman and Vogt [1, p. 15] that there is an infinite loop map $g: X \longrightarrow Y$ such that $g$ is a homotopy equivalence and $Y$ is the initial space of a spectrum in $\Omega S \cap W$. Combining this fact with $(v)$ of Theorem 4 , the remarks preceding that theorem, and (iii) of Theorem 6 , we see that if $f: x \rightarrow X^{\prime}$ is any infinite loop map between spaces of the homotopy type of countable CW-complexes, then there is a homotopy commutative 
diagram of infinite loop maps, of the form given in (I), such that $f^{\prime}$ is a perfect infinite loop map and $g$ and $g^{\prime}$ are homotopy equivalences.

Therefore nothing is lost for the purposes of weak homotopy theory if the notions of infinite loop spaces and maps are replaced by those of perfect infinite loop spaces and maps, and similarly for homotopy theory provided that we restrict attention to spaces of the homotopy type of countable CW-complexes.

The promised comparison of stable and unstable homotopy groups of infinite loop spaces is now an easy consequence of Proposition 1. In fact, if $\mathrm{Y}$ is an infinite loop space, say $Y=B_{0}$ where $B \in \Omega S$, then that proposition gives a map $\Phi_{\infty}(L M B): Q_{\infty} L_{0} M B \longrightarrow$ LMB in $L$, and Theorem 6 gives a map $n(M B): M B \longrightarrow I B$ in $I$. Define maps

$$
\mathrm{QY} \stackrel{\alpha}{\longrightarrow} \mathrm{QL}_{0} \mathrm{MB} \stackrel{\beta}{\longrightarrow} \mathrm{L}_{0} \mathrm{MB} \stackrel{\gamma}{\longleftarrow} \mathrm{Y}
$$

by $\alpha=Q \eta_{0}(M B), \beta=\Phi_{\infty, 0}(L M B)$, and $\gamma=n_{0}(M B), \quad \gamma$ is clearly a weak homotopy equivalence, and therefore so is $\alpha$ since Q: $T \longrightarrow T$ is easily verified to preserve weak homotopy equivalences. Since $\Phi_{\infty, 0}(\mathrm{IMB}) \cdot \Psi_{\infty}\left(L_{0} M B\right)$ is the identity map of $L_{0} M B, \beta_{*}$ is an epimorphism on homotopy. If $x \in T$, then $\Pi_{n}(Q X)=\Pi_{n}^{S}(X)$, the $n$th stable homotopy group of $x$. Therefore $\rho(Y)=\gamma_{*}^{-1} \beta_{*}^{\alpha}: \Pi_{*}(Q Y) \longrightarrow \Pi_{*}(Y)$ gives an epimorphism $\Pi_{*}^{S}(Y) \longrightarrow \Pi_{*}(Y)$. It is clear that if $f: Y \longrightarrow Y^{\prime}$ is any infinite loop map, then

$$
\rho\left(Y^{\prime}\right)(Q f)_{*}=f_{*} \rho(Y): \Pi_{n}^{S}(Y) \longrightarrow \Pi_{n}\left(Y^{\prime}\right) \text {. }
$$


It should be observed that the notions of infinite loop spaces and maps are not very useful from a categorical point of view since the composite of infinite loop maps need not be an infinite loop map. In fact, given infinite loop maps $\mathrm{f}: \mathrm{X} \longrightarrow \mathrm{Y}$ and $\mathrm{g}: \mathrm{Y} \longrightarrow \mathrm{Z}$, there need be no spectrum $\mathrm{B}$ with $B_{0}=Y$ which is simultaneously the range of a map of spectra giving $f$ and the domain of a map of spectra giving $g$. One can get around this by requiring infinite loop spaces to be topological monoids and using a classifying space argument to allow composition of maps, but this is awkward. These conditions motivate the use of $L$ in the definition of homology in section 1 .

The following application of our results, which will be used in the computation of $\mathrm{H}^{*}(B F)$, illustrates the technical convenience of the category $L$. Let $\widetilde{F}(n)=\operatorname{Hom}_{T}\left(S^{n}, S^{n}\right)$ and let $\widetilde{F}=\lim \widetilde{F}(n)$, where the limit is taken with respect to suspension of maps $S: \widetilde{F}(n) \longrightarrow \widetilde{F}(n+1) . \widetilde{F}(n)$ and $\widetilde{F}$ are topological monoids under composition of maps. If $x \in T$, define $\gamma: \Omega^{n_{X}} \times \widetilde{F}(n) \longrightarrow \Omega^{n} X$ by $\gamma(x, f)=\mu^{-n}\left(\mu^{n}(x) \cdot f\right)$, that is, with $\Omega^{n_{X}}$ identified with $\operatorname{Hom}_{T}\left(s^{0}, \Omega^{n} X\right)$, by the composite $\Omega^{n_{X}} \times \widetilde{F}(n) \stackrel{\mu^{n} \times 1}{\longrightarrow} \operatorname{Hom}_{T}\left(S^{n}, X\right) \times \widetilde{F}(n) \stackrel{\text { composition }}{\longrightarrow} \operatorname{Hom}_{T}\left(S^{n}, X\right) \stackrel{\mu^{-n}}{\longrightarrow} \Omega^{n} X$. This defines an operation of $\widetilde{F}(n)$ on $\Omega^{n} X$. Now let $B=\left\{B_{i}, f_{i}\right\} \in \Omega S$, and let $g_{i}: \Omega B_{i+1} \longrightarrow B_{i}$ be a homotopy inverse to $f_{i}$. Define homotopy equivalences $f^{n}: B_{0} \longrightarrow \Omega^{n_{B}}$ and $g^{n}: \Omega^{n_{B}} \longrightarrow B_{n}$ in the obvious inductive manner and define 


$$
\gamma_{n}=g^{n} \gamma\left(f^{n} \times 1\right): B_{0} \times \widetilde{F}(n) \longrightarrow B_{0} \cdot
$$

Observe that $\gamma_{n}$ fails to define an operation of $\widetilde{E}(n)$ on $B_{0}$ since the associativity condition $(x f) g=x(f g)$ is lost. of course, $\gamma_{n}$ coincides with $\gamma$ on $\Omega^{n} B_{n}$ if $B \in L$, and associativity is then retained. Now consider the following diagram:

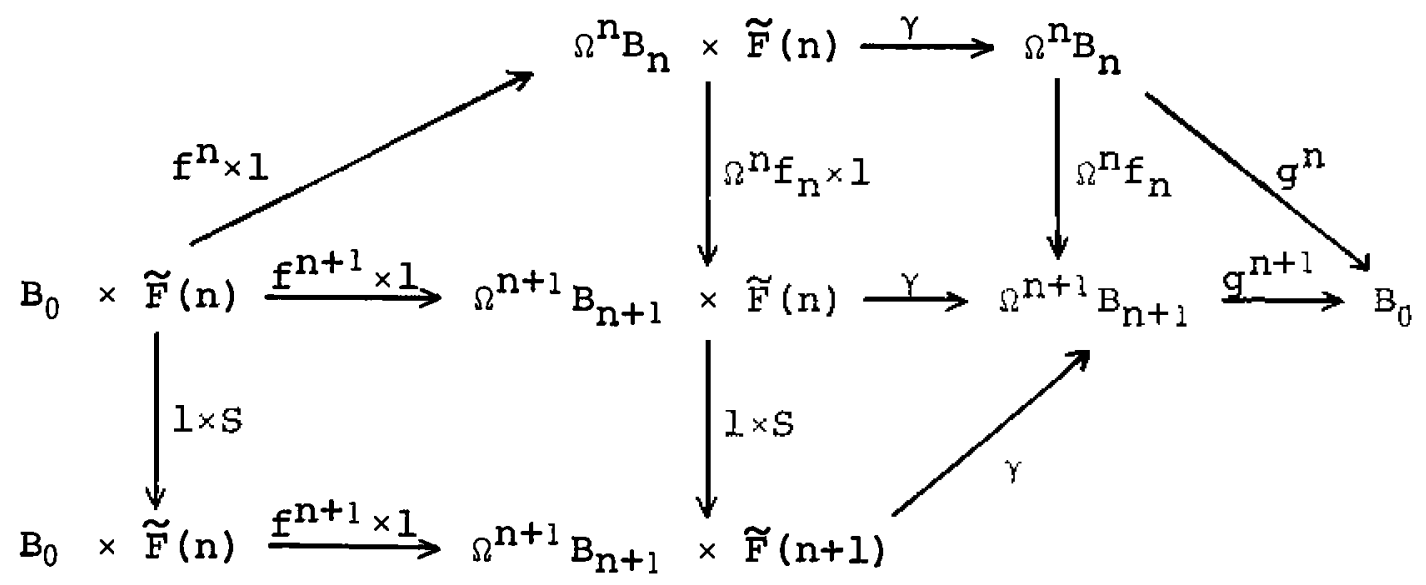

The left-hand triangle and square commute trivially. Clearly $\gamma$ is natural on $n$-fold loop maps, hence $\Omega^{n_{f_{n}} \gamma}=\gamma\left(\Omega^{n_{f}} \times 1\right)$. $\gamma(1 \times S)=\gamma$ since

$$
\mu^{-n}\left(\mu^{n}(x) f\right)=\mu^{-(n+1)} \mu\left(\mu^{n}(x) f\right)=\mu^{-(n+1)}\left(\mu^{n+1}(x) \text {. Sf }\right) \text {. }
$$

$g^{n}$ is homotopic to $g^{n+1} \Omega_{f^{n}}$, and if $B \in R$ and the $g_{i}$ are chosen retractions, then $g^{n}=g^{n+1} \Omega^{n_{f}}$. Thus if $B \in R$ we have $\gamma_{n}=\gamma_{n+1}(1 \times S)$ and we can define $\gamma=\stackrel{\lim }{\longrightarrow} \gamma_{n}: B_{0} \times \widetilde{F} \longrightarrow B_{0}$. Since the right-hand triangle is not transformed naturally by maps in $R$, the map $\gamma$ is not natural on $R$. For $B \in L$, the $f^{\prime} s$ and g's are the identity maps, and the diagram trivializes. Therefore, for each $B \in L$, we have an operation $\gamma: B_{0} \times \widetilde{F} \longrightarrow B_{0}$ and if $h: B \longrightarrow B^{\prime}$ is a map in $L$, then $h_{0}(x f)=h_{0}(x) f$ for $x \in B_{0}$ and $f \in \widetilde{F}$. 
Stasheff [unpublished] has generalized work of Dold and Lashof [2] to show that if a topological monoid $M$ operates on a space $x$, then there is a natural way to form an associated quasifibration $\mathrm{x} \longrightarrow \mathrm{Xx}_{\mathrm{M}} \mathrm{EM} \rightarrow \mathrm{BM}$ to the classifying principal quasifibration $\mathrm{M} \longrightarrow \mathrm{EM} \longrightarrow \mathrm{BM}$. As usual, let $\mathrm{F} \subset \widetilde{\mathrm{F}}$ consist of the homotopy equivalences of spheres. By restriction, if $B \in L$ and $Y=B_{0}$, we have an operation of $F$ on $Y$ and we can therefore form $\mathrm{Yx}_{\mathrm{F}} \mathrm{EF}$. Of course, this construction is natural on $L$.

Boardman and Vogt [1] have proven that the standard inclusions $U \subset O \subset P L \subset T O p \subset F$ are all infinite loop maps between infinite loop spaces with respect to the H-space structures given by Whitney sum (on F, this structure is weakly homotopic to the composition product used above). We now know that we can pass to $L$ and obtain natural operations of $F$ on (spaces homotopy equivalent to) each of these sub H-spaces $G$ of F. The same is true for their classifying spaces BG. Observe that the resulting operation of $F$ on $F$ is not equivalent to its product. (In fact, if $\phi \in B_{0}$ is the identity under the loop product of $\Omega B_{1}$, where $B \in L$, then $\phi f=\phi$ for all $f \in \widetilde{F}$ since composing any map with the trivial map gives the trivial map.) It would be of interest to understand the geometric significance of these operations by $F$ on its various sub H-spaces and of the spaces $G x_{F} E F$ and $B G x_{F} E F$.

I shall show elsewhere that, with mod $p$ coefficients, $\gamma_{*}: H_{*}(B) \otimes H_{*}(\widetilde{F}) \longrightarrow H_{*}(B)$ gives $H_{*}(B)$ a structure of Hopf 
algebra over $H_{*}(\widetilde{F})$ for $B \in L$ (and, a fortiori, for $B \in \Omega S$ ), where $\mathrm{H}_{*}(\mathrm{~B})=\mathrm{H}_{*}\left(\mathrm{~B}_{0}\right)$ as in section $1 . \mathrm{H}_{*}(\mathrm{~B})$ is also a Hopf algebra over the opposite algebra of the Steenrod algebra and over the Dyer-Lashof algebra, which is defined in terms of the homology operations introduced by Dyer and Lashof in [3]. These operations are all natural on $L$. The appropriate range category for $\mathrm{H}_{*}: L \longrightarrow$ ? is determined by specifying how these three types of homology operations commute, and, coupled with known information, these commutation formulas are all that is required to compute $\mathrm{H}^{*}(\mathrm{BF})$.

Finally, we observe that there is a natural way to extend our results of section 2 to unbounded spectra. Let $\bar{S}$ denote the category whose objects are sequences $\left\{B_{i}, f_{i} \mid i \in Z\right\}$ such that $\left\{B_{i}, f_{i} \mid i \geq 0\right\} \in S$ and $B_{i}=\Omega^{-i_{B_{0}}}$ and $f_{i}: B_{i} \longrightarrow \Omega B_{i+1}$ is the identity map for $i<0$. The maps in $\bar{S}$ are sequences $g=\left\{g_{i} \mid i \in z\right\}$ such that $\left\{g_{i} \mid i \geq 0\right\} \in S$ and $g_{i}=\Omega g_{i+1}$ if $i<0$. We have an obvious completion functor $C: S \rightarrow \bar{S}$ defined on objects by $C_{i} B=B_{i}$ if $i \geq 0$ and $C_{i} B=\Omega^{-i_{B_{0}}}$ if $i<0$, with $C_{i} f=f_{i}$ for $i \geq 0$ and $C_{i} f=1$ for $i<0$, and defined similarly on maps. $C$ is an isomorphism of categories with inverse the evident forgetful functor $\bar{S} \rightarrow S$. For each of our previously defined subcategories $D$ of $S$ define $\bar{D}$ to be the image of $D$ under $C$ in $\bar{S}$. $\bar{I}$ is of particular interest. Its objects and maps are sequences $\left\{B_{i} \mid i \in Z\right\}$ and $\left\{g_{i} \mid i \in z\right\}$ such that $B_{i}=\Omega B_{i+1}$ for all $i$ and $g_{i}=\Omega g_{i+1}$ for 
all i. Clearly all of the results of section 2 remain valid for the completed categories.

Our results show that any reasonable cohomology theory, by which we mean any cohomology theory determined by a spectrum B $\in \Omega \bar{S} \cap \mathbb{W}$, is isomorphic to a cohomology theory determined by a spectrum in $I \cap \mathbb{W}$ and that any transformation of such theories determined by a map $g: B \longrightarrow B^{\prime}$ in $\Omega \bar{S} \cap \pi$ is naturally equivalent to a transformation determined by a map in $I \cap \pi$. $\operatorname{Recall}$ that

$$
\mathrm{H}^{\mathrm{n}}(\mathrm{X}, \mathrm{A} ; \mathrm{B})=\operatorname{Hom}_{\mathrm{H} T}\left(\mathrm{X} / \mathrm{A}, \mathrm{B}_{\mathrm{n}}\right)
$$

defines the cohomology theory determined by $B \in \Omega \bar{S}$ on $C W$ pairs $(\mathrm{X}, \mathrm{A})$. Call such a theory connective if $\mathrm{H}^{\mathrm{n}}(\mathrm{P} ; \mathrm{B})=0$ for $\mathrm{n}>0$, where $P$ is a point. of course, $H^{-n}(P ; B)=\Pi_{0}\left(\Omega^{n_{B}}\right)=\Pi_{n}\left(B_{0}\right)$. Any infinite loop space $Y$ determines a connective (additive) cohomology theory since, by a classifying space argument, we can obtain $\mathrm{CB} \in \Omega \bar{S}$ such that $\mathrm{B}_{0}$ is homotopy equivalent to $\mathrm{Y}$ and $\pi_{0}\left(B_{n}\right)=0$ for $n>0$; according to Boardman and Vogt [1], anY such cohomology theory is so obtainable and determines $Y$ up to homotopy equivalence of infinite loop spaces. If $x \in T$, then $C Q_{\infty} \mathrm{X}$ determines a connective cohomology theory, since $C_{n} Q_{\infty} X=Q S^{n} X$ for $n>0$, and $H^{-n}\left(P ; C Q_{\infty} X\right)=\Pi_{n}(Q X)=\Pi_{n}^{S}(X)$ if $\mathrm{n} \geq 0$. In view of Proposition 1 , these theories play a privileged role among all connective cohomology theories, and an analysis of their properties might prove to be of interest. Observe that if $\mathrm{B} \in L$, then $\mathrm{C} \Phi_{\infty} \mathrm{B}: \mathrm{CQ}_{\infty} \mathrm{B}_{0} \longrightarrow \mathrm{CB}$ determines a natural transforma- 


$$
-478-
$$

tion of cohomology theories $H^{*}\left(X, A ; C Q_{\infty} B_{0}\right) \longrightarrow H^{*}(X, A, C B)$ and, if the theory determined by $\mathrm{CB}$ is connective, this transformation is epimorphic on the cohomology of a point. 


\section{BIBLIOGRAPHY}

[1] Boardman, J. M. and Vogt, R. M. "Homotopy Everything Hspaces", Mimeographed Notes, Math. Inst., Univ. of Warwick, (1968) .

[2] Dold, A. and Lashof, R., "Principal Quasifibrations and Fibre Homotopy Equivalence of Bundles", IZZ.J. Math., 3; 285-305, (1959).

[3] Dyer, E. and Lashof, R., "Homology of Iterated Loop Spaces", Amer. J. Math., 84; 35-88, (1962).

[4] Eilenberg, S. and MacLane, S., "Acyclic Models", Amer. J. Math., 75; 189-199, (1953).

[5] Hanner, 0., "Some Theorems on Absolute Neighborhood Retracts", Ark. Mat., 1, 389-408, (1950).

[6] Hurewicz, W. and Wallman, H., Dimension Theory, Princeton Univ. Press, (1961).

[7] Kuratowski, C., "Sur Les Espaces Localement Connexes et Péaniens en Dimension n", Fund. Math., 24; 269-287, (1935).

[8] Milgram, R. J., "Iterated Loop Spaces", Annals of Math., 84; 386-403, (1966).

[9] Milnor, J., "On Spaces Having the Homotopy Type of a CW-complex", Trans. A.M.S., 90; 272-280, (1959).

[10] Spanier, E. H., Algebraic Topology, McGraw-Hill, Inc., (1966).

[11] Swan, R. G., "The Homology of Cyclic Products", Trans. A.M.S., 95; 27-68, (1960). 